\title{
Development of Multilayer Focusing Mirror System for XFEL CDI Experiments of Biological Particles
}

Takahisa Koyama $^{1,2, *}$, Hirokatsu Yumoto ${ }^{1,2}$, Takashi Kimura ${ }^{3}$, Akihiro Suzuki ${ }^{3}$, Takashi Kameshima ${ }^{1,2}$, Yasumasa Joti $^{1,2}$, Kensuke Tono ${ }^{1,2}$, Naoya Tani ${ }^{3}$, Tatsuro Tachibana $^{3}$, Yusuke Konishi ${ }^{3}$, Yoshitaka Bessho $^{4}$, Yoshinori Nishino ${ }^{3}$, Makina Yabashi ${ }^{1,2}$, and Haruhiko Ohashi ${ }^{1,2}$

1. Japan Synchrotron Radiation Research Institute, 1-1-1 Kouto, Sayo-cho, Sayo-gun, Hyogo 679-5198, Japan

2. RIKEN SPring-8 Center, 1-1-1 Kouto, Sayo-cho, Sayo-gun, Hyogo 679-5148, Japan

3. Hokkaido University, Kita 21 Nishi 10, Kita-ku, Sapporo, Hokkaido 001-0021, Japan

4. Academia Sinica, 128 Academia Road, Section 2, Nankang, Taipei 11529, Taiwan

* Corresponding author, koyama@ spring8.or.jp

A single shot coherent diffractive imaging (CDI) using an X-ray free-electron laser (XFEL) enable us to visualize structures of a specimen without radiation damage owing to the femtosecond pulse nature. Living cells have been observed by pulsed coherent X-ray solution scattering (PCXSS) [1] using XFEL pulses from the SPring-8 Angstrom Compact free-electron LAser (SACLA). Although the resolution of a few tens nanometer has been achieved, there is a growing demand of resolution as high as single nanometer range. In order to meet the demand, we developed high-resolution CDI system employed multilayer focusing mirrors which has a high throughput and short focal lengths to further increase fluence at the sample plane.

The multilayer focusing mirrors were arranged with Kirkpatrick-Baez geometry. The surfaces of the substrates that made of synthetic silica glass with dimension of $80 \times 50 \times 30 \mathrm{~mm}^{3}$ were finished to suppress figure errors less than $1 \mathrm{~nm}$ (root-mean-square) by developing a special manufacturing process for the highly-curved surface. Designed parameters of the multilayer focusing mirrors were as follow: an incident angle of $25 \mathrm{mrad}$, a large spatial acceptance of more than $1 \mathrm{~mm}$ and short focal lengths of 190 $\mathrm{mm}$ and $100 \mathrm{~mm}$.

The multilayers were coated by DC magnetron sputtering method using a deposition system installed in the SPring-8 site. We adopted $\mathrm{Cr} / \mathrm{C}$ multilayer system which has wide spectral bandwidth and high peak reflectivity at a photon energy of $4 \mathrm{keV}$. The applied powers of the cathodes were $20 \mathrm{~W}$ for chromium and $60 \mathrm{~W}$ for carbon with process gas of argon in $\sim 0.1 \mathrm{~Pa}$. In the carbon deposition, two cathodes were used as co-deposition to increase deposition rate. The deposition rates were $1.6 \mathrm{~nm} / \mathrm{min}$ for chromium and $0.3 \mathrm{~nm} / \mathrm{min}$ for carbon. The number of multilayer period was set to be 30 and the ratio of $\mathrm{Cr}$ thickness/multilayer period was set to be 0.5 . The multilayer period lengths were laterally graded to $6.0 \sim 7.7 \mathrm{~nm}(5.1 \sim 8.5 \mathrm{~nm})$ in the upstream (downstream) mirror by controlling the substrate speed in front of the cathodes with a limited mask.

We confirmed XFEL irradiation tolerance of the multilayer coatings. Test pieces of the $\mathrm{Cr} / \mathrm{C}$ multilayer were irradiated with various irradiation fluences using $1 \mu \mathrm{m}$ XFEL focusing system [2] and dedicated irradiation chamber $[3,4]$ at BL3 of SACLA. The irradiation glancing angles were set to be the Bragg angles of the multilayers. Measured damage threshold fluences were one order of magnitude higher than unfocused beam fluence at the place of the CDI system. Therefore the $\mathrm{Cr} / \mathrm{C}$ multilayer system was applicable for focusing mirrors of the SACLA. 
We evaluated focusing performances of the mirrors at BL2 of SACLA. Beside the multilayer focusing mirrors, the CDI system was equipped with two sets of 4-blades slits, sample observation system, and sample scan stages as shown in Fig. 1. The sample observation system consists of a vacuum compatible objective lens with a fold mirror, and a visible light camera with a zoom lens tube arranged outside of the vacuum chamber. One can switch the two sets of 4-blades slits to the objective lens. The former was used as guard slits to reduce scattering X-rays on the area detector, the latter was used as sample positioning microscope to align a sample such as a micro-liquid enclosure array (MLEA) [1]. An X-ray area detector, a multiport charge coupled device (MPCCD) with octal sensor detector, closely located to the sample with a distance of $320 \mathrm{~mm}$, which covers a high-spatial frequency corresponding to a nominal spatial resolution of $2 \mathrm{~nm}$. We confirmed that a focused beam size of around $100 \mathrm{~nm}$ (FWHM) and a reflectivity of $\sim 55 \%$ (two bounce reflection) were achieved [5].

\section{References:}

[1] T. Kimura et al., Nat. Commun. 5 (2014) 3052.

[2] H. Yumoto et al., Nat. Photon. 7 (2013) 43.

[3] T. Koyama et al., Opt. Express 21 (2013) 15382.

[4] T. Koyama et al., Rev. Sci. Instrum. 87 (2016) 051801.

[5] The XFEL experiments were performed at the BL2 and BL3 of SACLA with the approval of the Japan Synchrotron Radiation Research Institute (JASRI) (Proposal No. 2015A8070, 2015B8067, 2016B8089, 2017A8085, 2017B8089, and 2018A8081).

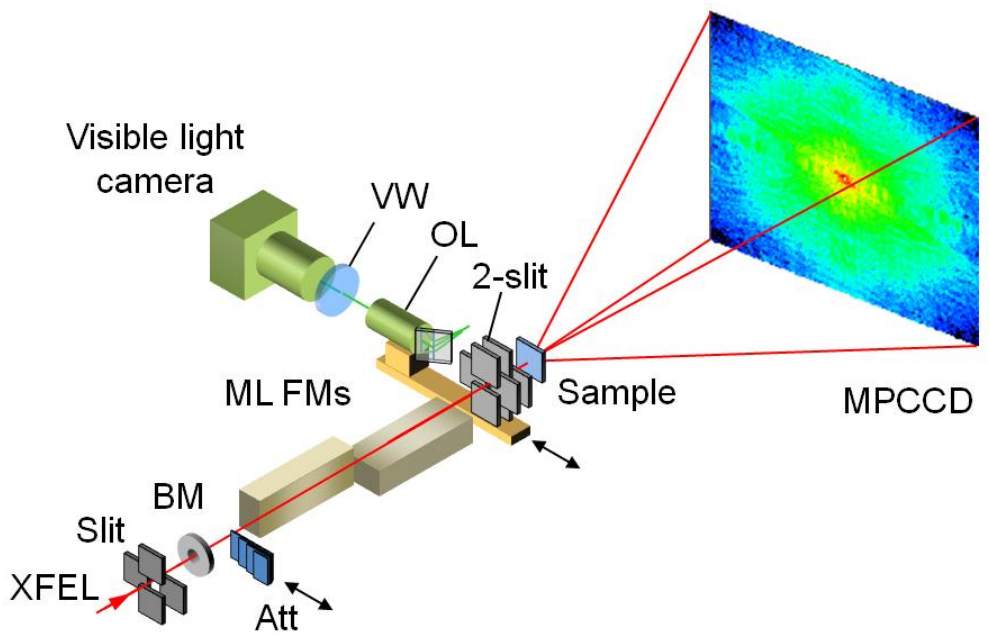

Figure. 1. Schematic drawing of the experimental setup. BM: Beam monitor, Att: Attenuator, ML FMs: Multilayer focusing mirrors, VW: Vacuum window, OL: Objective lens with a fold mirror, 2slit: two sets of 4-blades slits. 